\title{
Mutations in bdcA and valS correlate with quinolone resistance in wastewater Escherichia Coli
}

\author{
Negin Malekian ${ }^{1}$, Ali Al-Fatlawi ${ }^{1}$, Thomas U. Berendonk ${ }^{2}$ and Michael Schroeder ${ }^{{ }^{*}}$
}

\begin{abstract}
Single mutations can confer resistance to antibiotics. Identifying such mutations can help to develop and improve drugs. Here, we systematically screen for candidate quinolone resistance-conferring mutations. We sequenced highly diverse wastewater $E$. coli and performed a genome-wide association study (GWAS) correlating over 200,000 mutations against quinolone resistance phenotypes. We uncovered 13 statistically significant mutations including one located at the active site of the biofilm dispersal genes bdcA and six silent mutations in the aminoacyl-tRNA synthetase valS. The study also recovered the known mutations in the topoisomerases gyrA and parC.

In summary, we demonstrate that GWAS effectively and comprehensively identifies resistance mutations without a priori knowledge of targets and mode of action. The results suggest that bdcA and valS may be novel resistance genes with biofilm dispersal and translation as novel resistance mechanisms.
\end{abstract}

Keywords: E Coli; Quinolone; Antibiotic Resistance; Genome-Wide Association Study (GWAS)

\section{Background}

In the sixties, an impurity during the synthesis of the anti-malarial chloroquine led to the discovery of nalidixic acid $[1,2]$. Two years after its introduction to the market, resistances were observed, but it took another ten years before the drug's target and mechanism of action were understood [3]. Subsequently, improved derivatives of nalidixic acid were found, such as norfloxacin and ciprofloxacin and then levofloxacin. Today, there are over 20 fluoroquinolones on the market.

Generally, fluoroquinolones act by converting their targets, gyrase (gyrA) and topoisomerase IV (parC), into toxic enzymes that fragment the bacterial chromosome [4]. With the wide use of quinolones, however, bacteria developed resistances through several routes such as increased expression of efflux pumps, which transport drugs outside the bacterial cell, or horizontal gene transfer of resistance genes, whose gene products bind to the quinolone targets [4]. However, the most direct route to resistance is mutations in the drug targets gyrA and parC. Specifically, changes in the amino

\footnotetext{
${ }^{*}$ Correspondence: michael.schroeder@tu-dresden.de

${ }^{1}$ Biotechnology Center (BIOTEC), Technische Universität Dresden,

Tatzberg 47-49, 01307 Dresden, Germany

Full list of author information is available at the end of the article
}

acids Ser83 and Asp87 of gyrA and Ser80 of parC confer resistance $[4,5]$ to quinolones.

The discovery of these mutations was driven by a deep understanding of the mechanism of action of quinolones. Already over 50 years ago, Crumplin et al. suggested that "a comparative study of $[. .$.$] mu-$ tants and otherwise isogenic bacteria should facilitate identification of the hitherto unknown [...] target" [3], which was at the time not possible on a genome-wide scale. This changed with the advent of deep sequencing technology. Thus, we want to complement the original hypothesis-driven approach to understand resistance [3] with a hypothesis-free, high-throughput approach, in which we systematically evaluate the mutational landscape of resistant and susceptible bacteria.

Instead of investigating the quinolone targets in depth for resistance-conferring mutations, we screen entire bacterial genomes of many isolates and correlate them to patterns of the isolates' susceptibility and resistance. This approach termed genome-wide association study, GWAS, rose with the advent of deep sequencing and was initially applied to human genomes and disease phenotypes [6]. Recently, the success of human GWAS sparked interest in microbial GWAS [7, 8]. Genome-wide associations in bacteria are challenging, as clonal reproduction in bacteria leads to population 
stratification and a non-random association of alleles at different loci (linkage disequilibrium or LD) $[8,9]$.

E. coli's population structure is predominantly clonal, allowing the delineation of major phylogenetic groups, the largest being A (40\%), B2 (25\%), and B1 and D (both 17\%) [10]. Therefore, any model of a genome-wide association study in E. coli should accommodate these groups. Interestingly, the groups also relate to pathogenicity: Commensal E. coli, as e.g. found in human intestines, are more likely to belong to $\mathrm{A}$ and $\mathrm{B} 1$ and pathogenic to B2 and D.

Generally, E. coli genomes vary in size between 4000 to 5500 genes, of which only half are shared by all $E$. coli [11]. These genes, which are common to all E. coli, define the core-genome. It can be approximated as the intersection of genes present in a set of genomes. In contrast to the core-genome, the pan-genome is defined as the union of genes in a population. The E. coli pangenome exceeds 13000 genes and has possibly no limit due to their ability to absorb genetic material [11].

Parallel to the core and pan-genome, we coin the core and pan-variome. The former is defined as the intersection and the latter as the union of all mutations across all genomes. Mutations correlating with resistance will - by definition - not be part of the core-variome. Hence, it is important for a genome-wide association study that there is a significant gap in size between core and pan-variome.

A second major challenge besides population stratification is the dependencies of loci (linkage disequilibrium). The mutations in gyrA and parC correlate with each other, as they belong to the same resistance mechanism. However, following terminology from cancer biology, all of them are driver mutations, which cause clonal expansion in contrast to passenger mutations, which do not influence the fitness of a clone [12].

Driver mutations may impact clonal expansion directly by changing the amino acid sequence (nonsynonymous mutations) and thus protein structure or function. As an example, the gyrA and parC mutations are located at the drug's binding site and therefore influence binding. Driver mutations may also act indirectly as synonymous mutations without changes to the amino acid sequence. Synonymous mutations may have an effect on splicing, RNA stability, RNA folding, translation, or co-translational protein folding [13]. As an example, Kimchi et al. showed that a synonymous mutation in the multi-drug resistance gene MDR1 altered drug and inhibitor interactions. The authors argue that the reason may be a changed timing of co-translational folding and insertion into the membrane [14]. Thus, a genome-wide association study aiming to uncover novel resistance mechanisms should consider both non-synonymous and synonymous mutations, which are independent of already known mechanisms.

To date, it is not fully understood, how antibiotic resistance develops. It is ancient and inherent to bacteria [15] and can therefore be found in the natural environment. But with the wide use of antibiotics, major sources of resistant bacteria are clinics and wastewater [16]. In particular, the latter plays an important role, since treatment plants act as melting pots for bacteria of human, clinical, animal, and environmental origin [16]. The high genetic diversity of a clinical E. coli population was substantially exceeded by a wastewater population [17], which makes wastewater $E$. coli a suitable source for a GWAS analysis.

In summary, we aim to show that a bacterial genomewide association study can effectively and comprehensively identify targets relevant to antibiotic resistance. We aim to recover the known mutations in gyrA and parC together with novel candidate mutations. To maximise genomic diversity, we investigate wastewater E. coli. We employ a computational approach and implement variant calling on these genomes and then correlate the identified mutations against resistance levels of four quinolones covering first to third generation (nalidixic acid, norfloxacin, ciprofloxacin, and levofloxacin). We apply stringent filtering and cater for missing and rare data, population effects, and dependencies among mutations. Building on gyrA and parC mutations as controls, we expect to characterise the quantity and quality of the mutational resistance landscape. We will answer the question of whether there are resistance mutations beyond gyrA and parC and whether they may open new avenues for future drug discovery.

\section{Methods}

Sequencing and Phenotyping. Mahfouz et al. collected 1178 E. coli isolates from the inflow and outflow of the municipal wastewater treatment plant in Dresden, Germany. Based on representative resistance phenotypes, the authors selected 103 isolates for sequencing with Illumina MiSeq, 92 of which are available from NCBI's assembly database (PRJNA380388 : https:/ /www.ncbi.nlm.nih.gov/assembly/?term=PRJNA38 0388) and the rest by the authors. Phage and virus sequences were removed [17].

The unbiased sampling and selection of representative phenotypes were important for the subsequent GWAS analysis, which requires both resistant and susceptible isolates. The isolates were phenotyped using the agar diffusion method measuring the diameters of inhibition zone for 20 commonly prescribed antibiotics, including the four quinolones nalidixic acid, norfloxacin, ciprofloxacin, and levofloxacin [17]. 
bioRxiv preprint doi: https://doi.org/10.1101/2021.02.12.430739; this version posted February $13,2021$. The copyright holder for this preprint (which was not certified by peer review) is the author/funder, who has granted bioRxiv a license to display the preprint in perpetuity. It is made Malekian et al. available under aCC-BY-ND 4.0 International license.

Page 3 of 13

Variant Calling, Quality Control, and Functional Annotation. Reads were mapped onto E. coli K12 MG1655 with the Burrow-Wheeler Aligner (BWA) v0.7.12 and sorted with Picard v1.105. Variants were called using the genomic analysis toolkit GATK 4.1.1.0 [18] with E. coli K12 MG1655 as reference. We combined them into a single VCF file and re-genotyped them. Next, we filtered variants following standard protocols [19] and settings according to the GATK 4.1.1.0 website (for SNPs QD $<2.0$, QUAL $<$ 30.0 , or FS $>60.0$ and for INDELs QD $<2.0$, QUAL $<$ 30.0 , or FS $>200.0$ ). Variants with low genotype quality $(\mathrm{GQ}<20)$ and variants with $>15 \%$ of missing data were removed. After normalisation with BCFtools 1.7 [20], rare variants with minor allele frequency (MAF) $<5 \%$ were excluded with Pyseer 1.3.0. Finally, variants were functionally annotated using SnpEff $4.3 \mathrm{~T}$ [21].

Genome-Wide Association Study (GWAS). We performed a GWAS study by Pyseer 1.3.0 [22], using a generalized linear model for each variant. We built a phylogenetic tree from the VCF file with VCFkit 0.1.6 [23]. Using multidimensional scaling (MDS) on the distances in the phylogenetic tree, four outlier isolates were removed. For the remaining 99 isolates, we drew a scree plot for the eigenvalues of the MDS model and picked four components, which we used as covariates for the regression model to control for population structure. Finally, we calculated a Bonferronicorrected significance threshold for our GWAS analysis with pyseer.

Meta-analysis. We visualized GWAS results with quantile-quantile (QQ) and Manhattan plots using the $\mathrm{R}$ package qqman. ROC Curve and area under the curve (AUC) were calculated using the matplotlib and scikit-learn Python packages. We calculated the linkage disequilibrium (LD) between the loci of significant variants using PLINK v1.90b6.10 [24]. The R package LDheatmap [25] was used to visualize LD results. We applied and visualized MDS on the phylogenetic distances between the samples using the cmdscale and scatter3d functions from the stats and plot3d R packages, respectively. We drew a heatmap with dendrogram on the binary matrix of presence/absence of variants for different samples using the heatmap function from the $\mathrm{R}$ package stats.

3D structures. The 3D Structure of bdcA was retrieved from protein databank PDB (4PCV). The 3D structure of valS was retrieved from Swiss-model (based on PDB structure pdbid 1IVS). The 3d structures were visualized using PyMOL 2.2.0.
Conservation across other bacterial genomes. We retrieved the multiple sequence alignment ENOG50 1RQ0S for bdcA across all gammaproteobacteria from Eggnog 5.0 [26]. Residue 135 in the ungapped bdcA sequence was shifted to position 207 in the gapped multiple sequence alignment.

Conservation across other bacterial genomes. To check the frequency of bdcA G135S in other E. Coli genomes, we downloaded 1340 E. Coli genomes from NCBI (https://www.ncbi.nlm.nih.gov/) (accessed on 27th of October 2020) and identified the locus in each genome by searching for an exact match of the ten nucleotide long sequence ATTCACGGAG, which follows after the locus of the bdcA mutation and which is conserved across all the retrieved genomes.

\section{Results}

We aimed to identify mutations, which correlate with quinolone resistance. After extracting raw variants from 99 wastewater $E$. coli genomes, we proceeded in two steps: First, we reduced raw to high-quality and then high-quality to highly significant variants.

From raw to high-quality variants. From the genomes, we extracted 457,554 raw variants, which we subjected to five quality control steps resulting in 206,633 high-quality variants. Rare variants, which appear in less than $5 \%$ of isolates, led to the greatest reduction of mutations of nearly $50 \%$ (Table 1 ).

The pan- and core variome. For a genome-wide association study, it is vital that the mutations spread across the isolates. To characterise the distribution and diversity of the high-quality mutations, we computed the core and the pan-variome (see Figure 2). The corevariome reflects the number of variants shared by a given number of genomes. In contrast, the pan-variome consists of the union of all variants, thus reflecting the total diversity of variants present in all genomes. As expected, the pan-variome grows fast and the corevariome tails off fast. For 20 genomes, the pan-variome consists already of some 256,000 variants, while the core variome is reduced to some 600 variants. This means that there are only very few variants that are shared across many or even all of the genomes. Similarly, the graph for the pan-variome continually grows. Each added genome contributes new variants until the pan-variome reaches 413,283 variants (206,633 highquality plus 206,650 rare variants) in total. Overall, the distribution of variants is thus suitable for GWAS. 
bioRxiv preprint doi: https://doi.org/10.1101/2021.02.12.430739; this version posted February 13, 2021. The copyright holder for this preprint (which was not certified by peer review) is the author/funder, who has granted bioRxiv a license to display the preprint in perpetuity. It is made Malekian et al. available under aCC-BY-ND 4.0 International license.

Page 4 of 13

From high-quality to highly significant variants Next, we carried out a GWAS study correlating the high-quality variants against resistance levels of the four quinolones investigated (nalidixic acid, norfloxacin, ciprofloxacin, and levofloxacin). Two aspects were important: We wanted to control the population structure and ensure the independence of the novel mutations from the known resistance-conferring mutations.

To assess the control of the study over the population structure, we plotted p-values expected under randomness against observed p-values (see QQ plots in Figure 3). The plots confirm that the correction for population structure was satisfactory, as a deviation from the null hypothesis (the identity line) is only evident at the tail of the plots.

Next, we visualized the results of the GWAS using Manhattan plots, which reveal that there are some highly significant variants passing the rigorous Bonferroni-corrected p-value (the horizontal line). To confirm the level of significance, we evaluated how well these variants predict resistance. To this end, we plotted a receiver operating characteristic (ROC) curve and calculated the area under the curve (AUC) as a measure of predictive performance. The AUC for most of the significant variants was above $90 \%$ (see Figure 3) reflecting that the identified variants very accurately predict resistance.

Summary statistics of the GWAS analysis. In total, we obtained 13 highly significant variants, three in gyrA and parC and ten novel candidate variants in the five genes bdcA, valS, lptG, lptF, and ivy. The variant in bdcA leads to an amino acid change, while the remaining nine do not.

Across all four quinolones, the mutations in gyrA and parC ranked highest thus confirming the validity of the approach taken (Table 2). As shown in the table, the frequency and effect sizes of the novel candidate variants are on a par with the positive controls. This means that the existence of an effect (p-value) and the size of the effect (beta) are both given. While all variants pass the Bonferroni-corrected p-value threshold (5.21E-07), the positive controls exceed it very substantially (Table 3 ).

Novel candidate variants are independent of controls. To check the independence of the significant variants from one another, we measured the linkage disequilibrium (LD) for the loci of these variants (see Figure 4). The known quinolone resistanceconferring variants, gyrA S83L, gyrA D87N, and parC S80I are in LD. They are located at the drugs' binding sites to gyrA and parC and ensure the correct function of the gene products despite treatment.
The known resistance-conferring variants are not in LD with the ten novel loci, which suggests that they confer resistance by a different mechanism from gyrA and parC. Among the novel loci, there are dependencies. In particular, the non-synonymous variant in bdcA is in LD with synonymous mutations in valS. This may mean that these novel variants act in a shared mechanism, which raises the question of whether the biological functions of the novel loci can be linked to antibiotic resistance.

Biological function of bdcA. The bdcA gene plays a role in biofilm dispersal $[27,28]$ and generally, biofilm formation increases antimicrobial resistance $[29,30]$. It could be hypothesised that a variant in this gene disrupts biofilm dispersal and leads to biofilm formation and resistance. However, while this may happen in nature, it is unclear whether this effect is also present in the disk diffusion assay underlying the present data. This gene is present in nearly all isolates (85-90\% in our data and NCBI data), which means that is close to being a core gene, but that it is not essential for survival.

Biological function of valS. The valS gene product is an aminoacyl-tRNA synthetase (aaRS), which charges tRNA encoding valine with the valine amino acid. The aaRS enzymes are promising targets for antimicrobial development [31, 32] as targeting them can inhibit the translation process, cell growth, and finally cell viability. Although aaRS enzymes are not known as direct quinolone targets, there is evidence that non-synonymous mutations in aaRS enzymes increase ciprofloxacin resistance by upregulating the expression of efflux pumps [33]. In our data, we found synonymous valS mutations for ciprofloxacin just below the p-value cut-off. For levofloxacin and norfloxacin, they were above the cut-off. valS provides a very basic function and is a core gene present in all isolates.

Biological function of ivy. The gene product of ivy is a strong inhibitor of lysozyme C. Expression of ivy protects porous cell-wall $E$. coli mutants from the lytic effect of lysozyme, suggesting that it is a response against the permeabilizing effects of the innate vertebrate immune system. As such, ivy acts as a virulence factor for a number of gram-negative bacteria-infecting vertebrates [34].

Biological function of lptG and lptF. The gene products of lptG and lptF are part of the ABC transporter complex LptBFG involved in the translocation of lipopolysaccharide from the inner membrane to the outer membrane. Thus, there is no direct connection 
bioRxiv preprint doi: https://doi.org/10.1101/2021.02.12.430739; this version posted February 13, 2021. The copyright holder for this preprint (which was not certified by peer review) is the author/funder, who has granted bioRxiv a license to display the preprint in perpetuity. It is made Malekian et al. available under aCC-BY-ND 4.0 International license.

Page 5 of 13

to antibiotic resistance, however, the link to transport is in line with other resistance mechanisms such as increased expression of efflux pumps [35].

Structural Analysis of bdcA and valS. To shed more light on the possible causality of the GWAS candidate variants, we explored their protein structures (Figure 5). The variant Gly135Ser in bdcA is in the vicinity of the active site residues Ser132 and Tyr146 [27]. Serine is bigger than glycine and it may influence a loop formed by the residues 136-144 and thus regulate the active site, which may influence biofilm dispersal.

In valS, the identified variants are synonymous and thus have no direct impact on the structure of the protein. However, for some loci, there were nonsynonymous variants such as e.g. D452E. Therefore, we wanted to understand, where the valS mutations are located in the 3D structure. Figure 5 shows the structure of a model for valS in E. coli, which is generated by Swiss-model based on a template in Thermus thermophilus. The model reveals that the valS mutations are on the surface of the protein.

Variant bdcA G135S wrt. other antibiotics, other $E$. coli, and other bacterial sequences. For the non-synonymous variant bdcA G135S, we wanted to understand whether its role in antibiotic resistance is limited to quinolones or not. For 16 other antibiotics, [17] there are variants, which significantly correlated with resistance (data not shown). For all antibiotics but tobramycin, the bdcA mutation is not significant. This suggests, that bdcA G135S may act independently of fluoroquinolone, which would be consistent with biofilm formation being a general mechanism independent of fluoroquinolone.

Next, we wanted to know whether the prevalence of bdcA G135S in our data is representative of other $E$. coli genomes. In 1340 complete E. coli genomes available from the NCBI, we could find the bdcA gene in 1209 genomes and bdcA G135S in 24. Thus, about $2 \%$ of genomes carry this mutation, which is slightly less, but comparable to the $5 \%$ present in our data.

$\mathrm{BdcA}$ is present in other bacteria. We investigated gammaproteobacteria, which comprise pseudomonadaceae besides enterobacteria. We analysed 152 bdcA sequences retrieved from Eggnog 5.0 and found alanine most frequently (65\%) and glycine less frequently (24\%). Serine appeared in $2 \%$ of the species, which may mean that the resistance mechanism is not limited to E. coli.

Phylogenetic groups. A key ingredient of the GWAS model is the population structure. We ap- plied dimension reduction and hierarchical clustering to isolates represented as high-dimensional binary vectors, where each dimension corresponds to one of the 206,633 mutations. We identified four clusters (Figure 6), which broadly correspond to phylogenetic groups A, B1, B2, and D. Thus, our GWAS model correctly caters for the main E. coli lineages.

\section{Discussion and Conclusion}

It took over a decade to move from the discovery of nalidixic acid to the discovery of its target and mechanism of action. Here, we have shown that sequencing and phenotyping data of a small number of genomes from a single site are sufficient for a GWAS model to reveal the quinolone targets with a very high statistical significance. Furthermore, the GWAS model revealed ten new mutations, which correlate significantly with quinolone resistance. A key to the success of the GWAS model was an unbiased sampling of isolates, which contained resistant and susceptible isolates.

The most promising mutation is G135S in the biofilm dispersal gene bdcA, which is present in nearly all isolates, but which is not essential for $E$. coli survival [36]. Mapping the bdcA mutation onto a protein structure of bdcA revealed its location on the surface of the protein and close to the active site. Hence, this suggests an impact on enzymatic activity, which may influence biofilm dispersion and hence indirectly relate to antibiotic resistance. In fact, $\mathrm{Ma}$ et al. could show that $E$. coli bdcA controls biofilm dispersal in Pseudomonas aeruginosa [37], which were the most abundant gammaproteobacteria containing bdcA in our analysis. This indicates that mutations in $E$. coli bdcA may act indirectly on antibiotic resistance. If consequently, bdcA emerges as a novel drug target, then the next steps in drug development could target the active site with residues S132 and Y146, which are in direct proximity to the mutation bdcA G135S. Importantly, bdcA G135S is a novel candidate resistance mutation as it is not in $\mathrm{LD}$ with the known mutations in gyrA and parC.

We found bdcA G135S in 5\% of the analysed genomes, which appears in line with a prevalence of $2 \%$ in 1209 other E. coli genomes obtained from the NCBI. We also checked the presence of these mutations in other gammaproteobacteria and revealed that bdcA is present and well conserved, but that the mutation appears specific to E. coli. Furthermore, we also checked whether bdcA G135S correlates with resistance to non-quinolone antibiotics. This was the case for tobramycin, an aminoglycoside, but not for all other examined antibiotics. Isolates with the bdcA G135S mutation belonged to the phylogenetic group A, which is less likely to contain pathogenetic isolates. 
Phylogroup A is equally abundant in human faeces and wastewater [38], which may point to an origin of the mutation in a human rather than a natural environment.

Besides bdcA G135S, we found nine mutations, which are synonymous, whose mechanism of action is likely to be indirect. Most interesting are the abundant mutations in the aminoacyl-tRNA synthetase valS, which has an essential role in protein synthesis and which is part of the core-genome and is therefore present in all isolates. Furthermore, it is classified as an essential gene [36]. It may be a suitable drug target [39] due to their evolutionary divergence between prokaryotic and eukaryotic enzymes, high conservation across different bacterial pathogens, as well as solubility, stability, and ease of purification. However, since the mutations in valS were synonymous, they will not exert a direct structural or functional effect on their gene product but may act indirectly.

In summary, bdcA G135S and the discovered silent mutations are statistically significant correlating with quinolone resistance in wastewater $E$. coli. They appear to be mostly specific to $E$. coli and to quinolones and independent of known resistance-conferring mutations. Further research is needed to corroborate the correlation between these mutations and quinolone resistance and to shed light on the molecular mechanism leading to resistance 
bioRxiv preprint doi: https://doi.org/10.1101/2021.02.12.430739; this version posted February 13, 2021. The copyright holder for this preprint (which was not certified by peer review) is the author/funder, who has granted bioRxiv a license to display the preprint in perpetuity. It is made

Wastewater

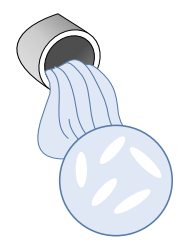

E. Coli
Resistance Phenotyping

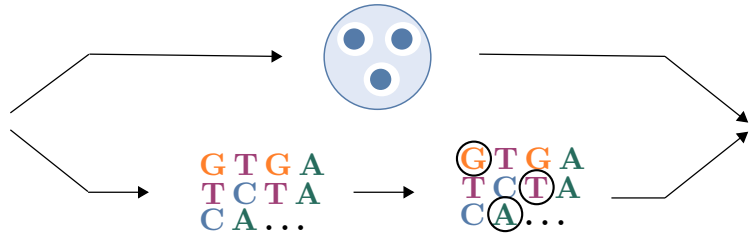

Sequencing Variant Calling

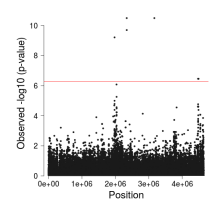

GWAS
bdcA Mutation(s)

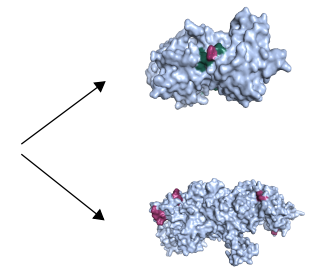

valS Mutation(s)

Figure 1: Wastewater E. Coli were phenotyped and sequenced. Variants were called and correlated to quinolone resistance in a GWAS study resulting in novel candidate resistance mutations.

Table 1: Quality control (QC): Reduction of some 457.000 raw variants to 206.633 high-quality variants. Rare variants (MAF) is the main filter.

\begin{tabular}{lrr}
\hline Step & Change & Mutations \\
\hline 1. Variant calling & & 457,554 \\
2. Hard filters & $-2 \%$ & 449,017 \\
3. GQ filter and missingness & $-15 \%$ & 382,922 \\
4. Normalisation by allele & $+8 \%$ & 413,283 \\
5. Minor allele frequency (MAF) & $-50 \%$ & 206,633 \\
\hline
\end{tabular}

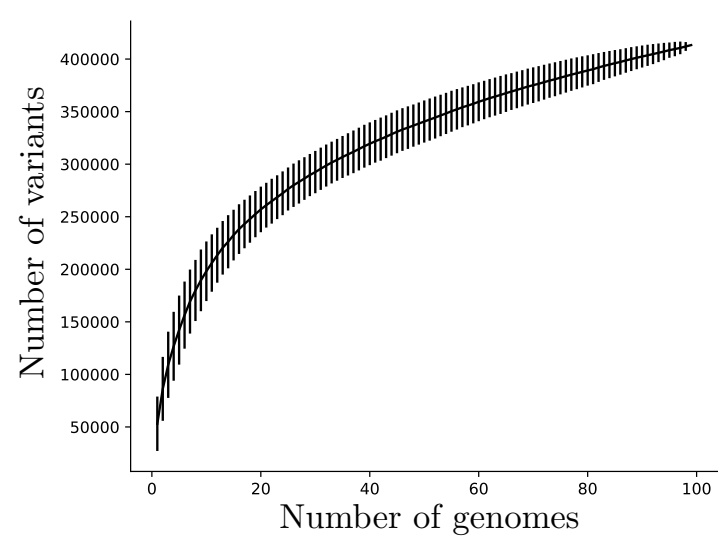

a) Pan-variome

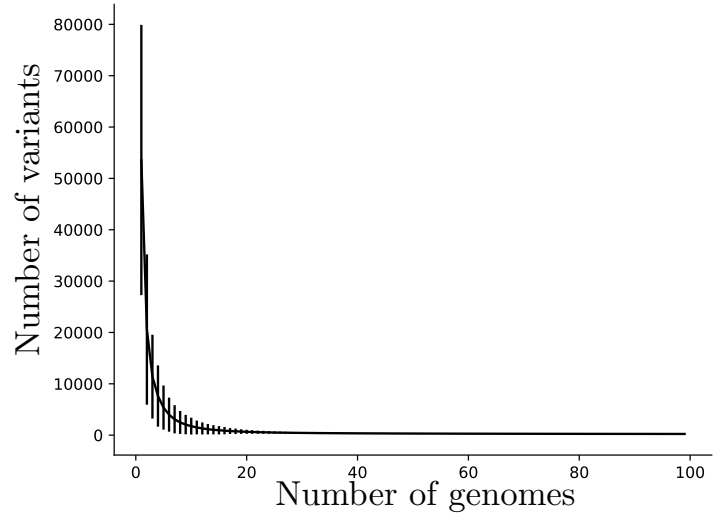

b) Core-variome

Figure 2: Pan-variome (union of variants) and core-variome (intersection of variants) of 206,633 high-quality and 206,650 rare variants (413,283 in total). Most variants appear only in a few of the isolates. 
bioRxiv preprint doi: https://doi.org/10.1101/2021.02.12.430739; this version posted February 13, 2021. The copyright holder for this preprint (which was not certified by peer review) is the author/funder, who has granted bioRxiv a license to display the preprint in perpetuity. It is made available under aCC-BY-ND 4.0 International license.
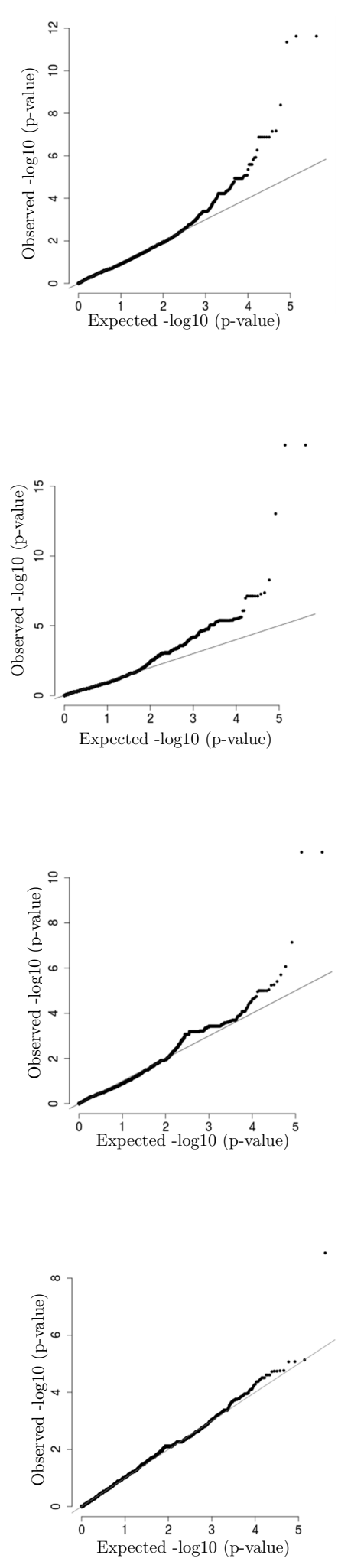

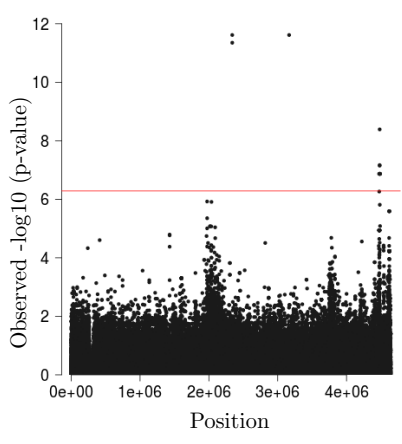

a) Levofloxacin

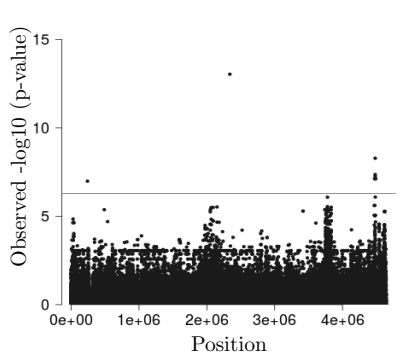

b) Norfloxacin
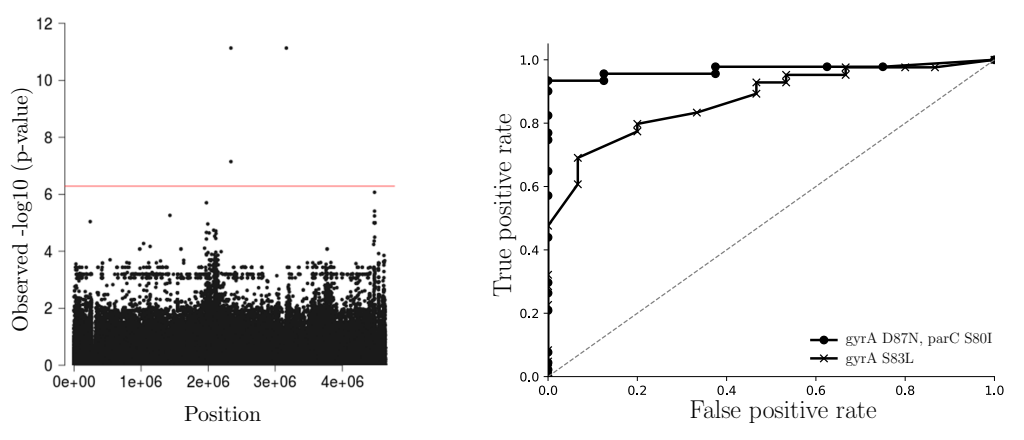

c) Ciprofloxacin
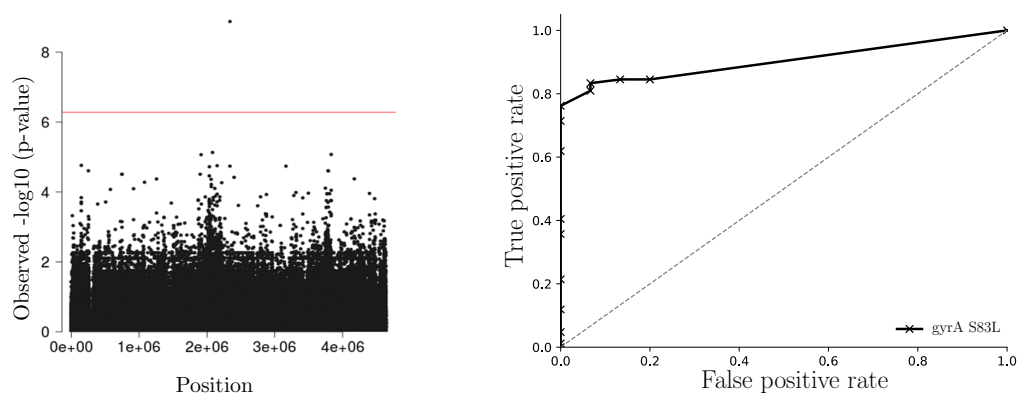

d) Nalidixic acid
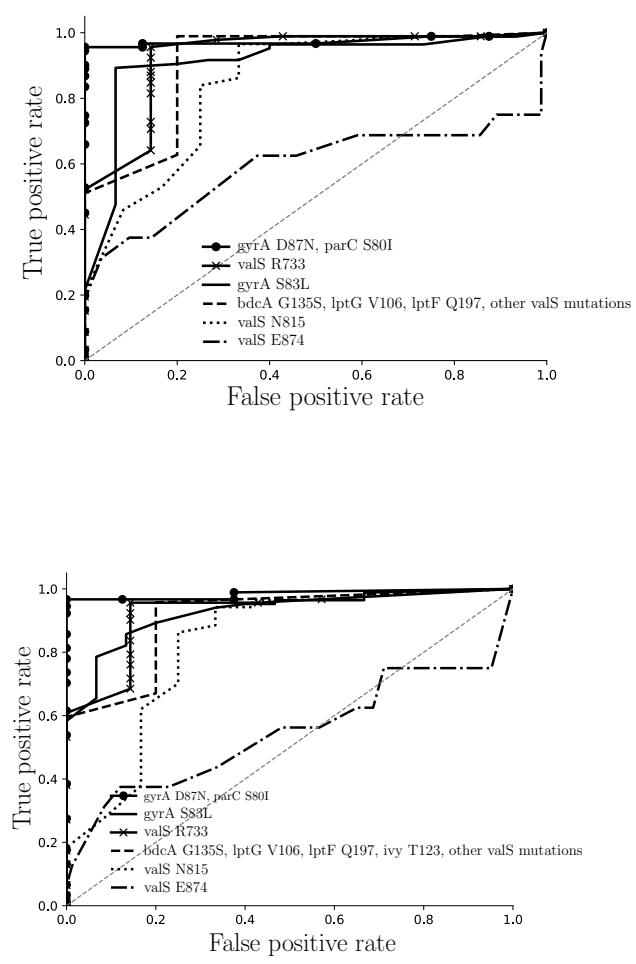

$$
\text { (1) }
$$

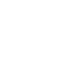


bioRxiv preprint doi: https://doi.org/10.1101/2021.02.12.430739; this version posted February $13,2021$. The copyright holder for this preprint (which was not certified by peer review) is the author/funder, who has granted bioRxiv a license to display the preprint in perpetuity. It is made

Table 2: Mutations significantly correlating with quinolone resistance. Freq. is the relative frequency among isolates and Beta the effect size. Effect size is similar for all, p-values differ.

\begin{tabular}{|c|c|c|c|c|c|c|c|c|c|c|}
\hline Quinolone & Position & Allele & Gene & Effect & Freq. & Beta & SE & Call rate & P-value & AUC \\
\hline \multirow{12}{*}{ Levofloxacin } & 3165735 & $A$ & parC & S80I & 0.08 & -1.56 & 0.20 & $100 \%$ & $2.43 \mathrm{E}-12$ & $97 \%$ \\
\hline & 2339162 & $\mathrm{~T}$ & gyrA & D87N & 0.08 & -1.56 & 0.20 & $100 \%$ & 2.43E-12 & $97 \%$ \\
\hline & 2339173 & A & gyrA & S83L & 0.15 & -1.20 & 0.16 & $99 \%$ & 4.47E-12 & $91 \%$ \\
\hline & 4473651 & $\mathrm{~T}$ & bdcA & G135S & 0.05 & -1.58 & 0.29 & $90 \%$ & $1.35 \mathrm{E}-07$ & $91 \%$ \\
\hline & 4481639 & A & valS & R733 & 0.07 & -1.15 & 0.24 & $100 \%$ & $4.09 \mathrm{E}-09$ & $93 \%$ \\
\hline & 4481393 & A & vals & N815 & 0.12 & -1.11 & 0.20 & $100 \%$ & $6.79 \mathrm{E}-08$ & $84 \%$ \\
\hline & 4481216 & $\mathrm{~T}$ & valS & E874 & 0.16 & -1.61 & 0.29 & $100 \%$ & $7.09 \mathrm{E}-08$ & $59 \%$ \\
\hline & 4482482 & A & valS & D452 & 0.05 & -1.58 & 0.29 & $100 \%$ & $1.35 \mathrm{E}-07$ & $91 \%$ \\
\hline & 4482443 & A & vals & V465 & 0.05 & -1.58 & 0.29 & $100 \%$ & $1.35 \mathrm{E}-07$ & $91 \%$ \\
\hline & 4482440 & $\mathrm{~T}$ & ValS & L466 & 0.05 & -1.58 & 0.29 & $100 \%$ & $1.35 \mathrm{E}-07$ & $91 \%$ \\
\hline & 4486808 & A & IptF & Q197 & 0.05 & -1.58 & 0.29 & $100 \%$ & $1.35 \mathrm{E}-07$ & $91 \%$ \\
\hline & 4487635 & A & IptG & V106 & 0.05 & -1.58 & 0.29 & $100 \%$ & $1.35 \mathrm{E}-07$ & $91 \%$ \\
\hline \multirow{13}{*}{ Norfloxacin } & 3165735 & $A$ & parC & S80I & 0.08 & -2.29 & 0.22 & $100 \%$ & $1.10 \mathrm{E}-18$ & $98 \%$ \\
\hline & 2339162 & $\mathrm{~T}$ & gyrA & D87N & 0.08 & -2.29 & 0.22 & $100 \%$ & $1.10 \mathrm{E}-18$ & $98 \%$ \\
\hline & 2339173 & A & gyrA & S83L & 0.15 & -1.59 & 0.19 & $99 \%$ & $9.25 \mathrm{E}-14$ & $93 \%$ \\
\hline & 4473651 & $\mathrm{~T}$ & bdcA & G135S & 0.05 & -2.01 & 0.36 & $90 \%$ & 7.56E-08 & $91 \%$ \\
\hline & 4481639 & $A$ & valS & R733 & 0.07 & -1.85 & 0.30 & $100 \%$ & $5.24 \mathrm{E}-09$ & $92 \%$ \\
\hline & 4481216 & $\mathrm{~T}$ & valS & E874 & 0.16 & -2.03 & 0.35 & $100 \%$ & 4.36E-08 & $55 \%$ \\
\hline & 4481393 & A & valS & N815 & 0.12 & -1.39 & 0.25 & $100 \%$ & $5.40 \mathrm{E}-08$ & $82 \%$ \\
\hline & 4482482 & A & valS & D452 & 0.05 & -2.01 & 0.36 & $100 \%$ & $7.56 \mathrm{E}-08$ & $91 \%$ \\
\hline & 4482443 & A & valS & V465 & 0.05 & -2.01 & 0.36 & $100 \%$ & $7.56 \mathrm{E}-08$ & $91 \%$ \\
\hline & 4482440 & $\mathrm{~T}$ & valS & L466 & 0.05 & -2.01 & 0.36 & $100 \%$ & $7.56 \mathrm{E}-08$ & $91 \%$ \\
\hline & 4486808 & A & IptF & Q197 & 0.05 & -2.01 & 0.36 & $100 \%$ & $7.56 \mathrm{E}-08$ & $91 \%$ \\
\hline & 4487635 & A & lptG & V106 & 0.05 & -2.01 & 0.36 & $100 \%$ & $7.56 \mathrm{E}-08$ & $91 \%$ \\
\hline & 240711 & $\mathrm{~T}$ & ivy & $\mathrm{T} 123$ & 0.05 & -2.00 & 0.36 & $100 \%$ & $1.04 \mathrm{E}-07$ & $91 \%$ \\
\hline \multirow{3}{*}{ Ciprofloxacin } & 3165735 & $A$ & parC & S80I & 0.08 & -1.90 & 0.25 & $100 \%$ & $7.37 \mathrm{E}-12$ & $97 \%$ \\
\hline & 2339162 & $\mathrm{~T}$ & gyrA & D87N & 0.08 & -1.90 & 0.25 & $100 \%$ & 7.37E-12 & $97 \%$ \\
\hline & 2339173 & A & gyrA & S83L & 0.15 & -1.22 & 0.22 & $99 \%$ & 7.13E-08 & $87 \%$ \\
\hline Nalidixic acid & 2339173 & $A$ & gyrA & S83L & 0.15 & -1.57 & 0.24 & $99 \%$ & $1.32 \mathrm{E}-09$ & $90 \%$ \\
\hline
\end{tabular}

Table 3: Ranking of mutations significantly correlating with quinolone resistance.

\begin{tabular}{rlllrrrr} 
& & & & Levofloxacin & Norfloxacin & Ciprofloxacin & Nalidixic acid \\
\hline Position & Allele & Gene & Effect & Rank/P-value & Rank/P-value & Rank/P-value & Rank/P-value \\
\hline 3165735 & A & parC & S80I & $1 / 2.43 E-12$ & $1 / 1.10 E-18$ & $1 / 7.37 E-12$ & $7 / 1.82 E-05$ \\
2339162 & T & gyrA & D87N & $1 / 2.43 E-12$ & $1 / 1.10 E-18$ & $1 / 7.37 E-12$ & $7 / 1.82 E-05$ \\
2339173 & A & gyrA & S83L & $3 / 4.47 E-12$ & $3 / 9.25 E-14$ & $3 / 7.13 E-08$ & $1 / 1.32 E-09$ \\
4473651 & T & bdcA & G135S & $7 / 1.35 E-07$ & $7 / 7.56 E-08$ & $10 / 1.00 E-05$ & $152 / 6.44 E-04$ \\
\hline 4481639 & A & valS & R733 & $4 / 4.09 E-09$ & $4 / 5.24 E-09$ & $4 / 8.50 E-07$ & $182 / 8.00 E-04$ \\
4481393 & A & valS & N815 & $5 / 6.79 E-08$ & $6 / 5.40 E-08$ & $6 / 3.90 E-06$ & $405 / 2.14 E-03$ \\
4481216 & T & valS & E874 & $6 / 7.09 E-08$ & $5 / 4.36 E-08$ & $8 / 5.75 E-06$ & $162 / 6.67 E-04$ \\
4482482 & A & valS & D452 & $7 / 1.35 E-07$ & $7 / 7.56 E-08$ & $10 / 1.00 E-05$ & $152 / 6.44 E-04$ \\
4482443 & A & valS & V465 & $7 / 1.35 E-07$ & $7 / 7.56 E-08$ & $10 / 1.00 E-05$ & $152 / 6.44 E-04$ \\
4482440 & T & valS & L466 & $7 / 1.35 E-07$ & $7 / 7.56 E-08$ & $10 / 1.00 E-05$ & $152 / 6.44 E-04$ \\
4486808 & A & IptF & Q197 & $7 / 1.35 E-07$ & $7 / 7.56 E-08$ & $10 / 1.00 E-05$ & $152 / 6.44 E-04$ \\
4487635 & A & IptG & V106 & $7 / 1.35 E-07$ & $7 / 7.56 E-08$ & $10 / 1.00 E-05$ & $152 / 6.44 E-04$ \\
240711 & T & ivy & T123 & $68 / 4.69 E-05$ & $13 / 1.04 E-07$ & $9 / 9.07 E-06$ & $395 / 2.08 E-03$
\end{tabular}


bioRxiv preprint doi: https://doi.org/10.1101/2021.02.12.430739; this version posted February 13, 2021. The copyright holder for this preprint (which was not certified by peer review) is the author/funder, who has granted bioRxiv a license to display the preprint in perpetuity. It is made available under aCC-BY-ND 4.0 International license.

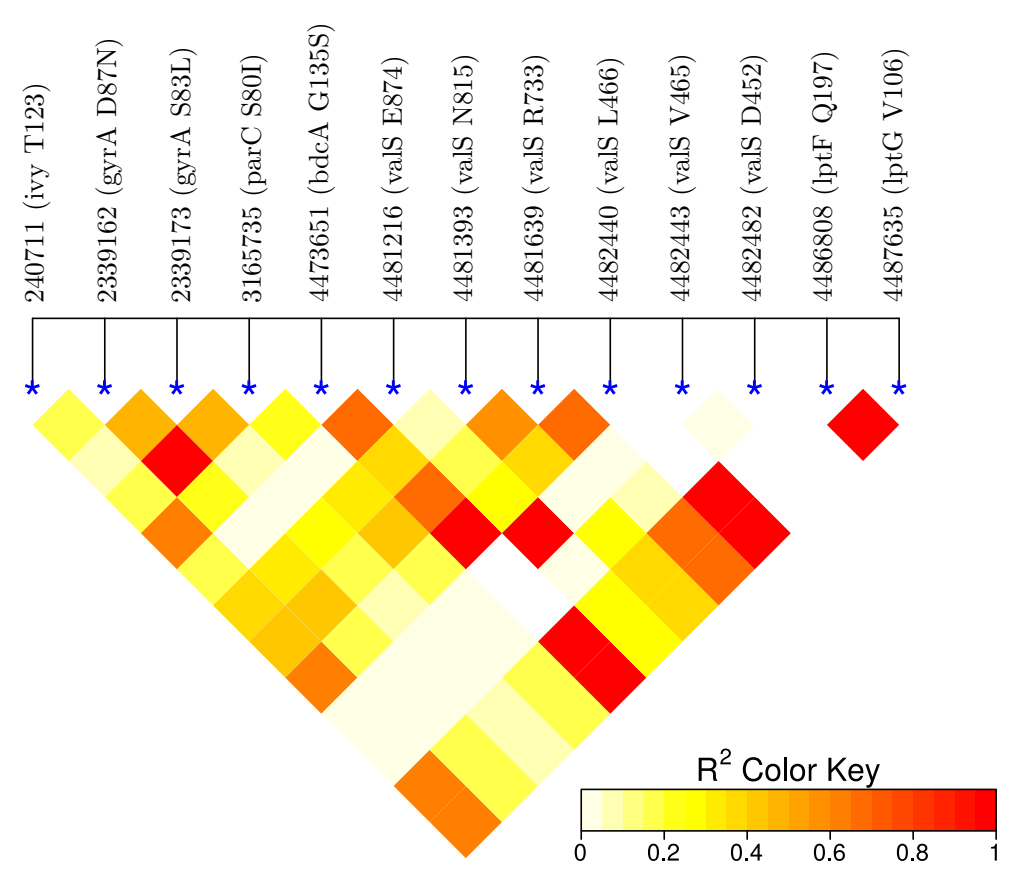

Figure 4: Linkage disequilibrium. High values (red) indicate a dependence of the loci. As expected, the loci in gyrA and parC are in linkage disequilibrium. Importantly, they are not in LD with the remaining novel candidate loci. Interestingly, there is some dependence within the novel loci, in particular, bdcA is in LD with valS.

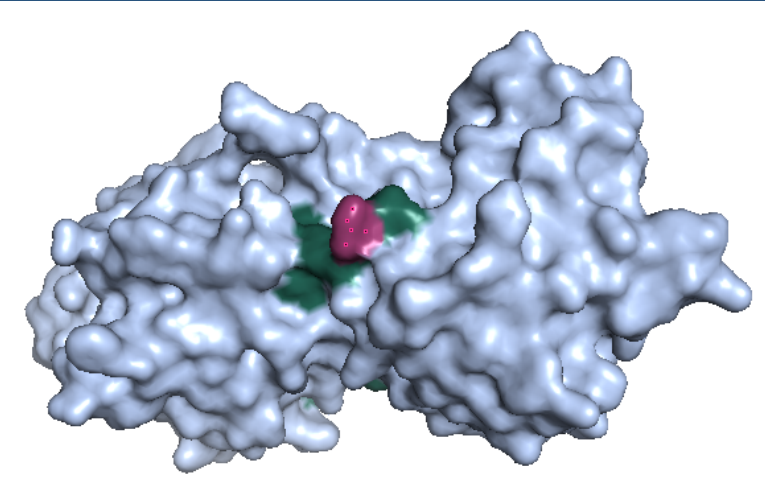

a) bdcA

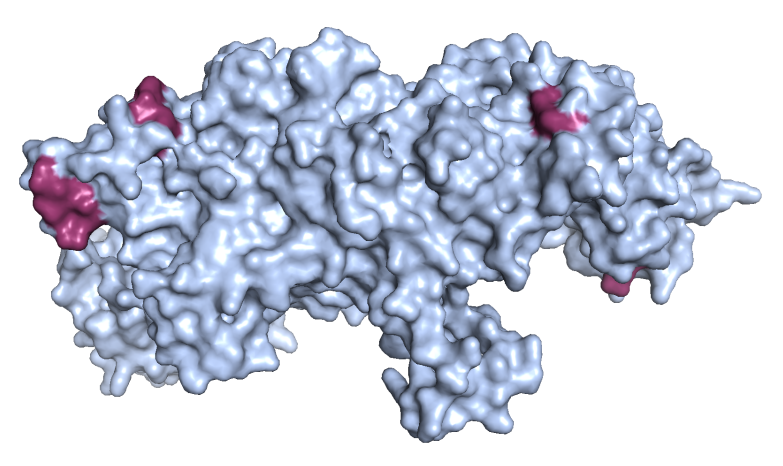

b) valS

Figure 5: 3D structures of bdcA and valS. Significant mutations (red) are at the surface and bdcA G135S is near the active site (green). 
bioRxiv preprint doi: https://doi.org/10.1101/2021.02.12.430739; this version posted February 13,2021 . The copyright holder for this preprint (which was not certified by peer review) is the author/funder, who has granted bioRxiv a license to display the preprint in perpetuity. It is made available under aCC-BY-ND 4.0 International license.

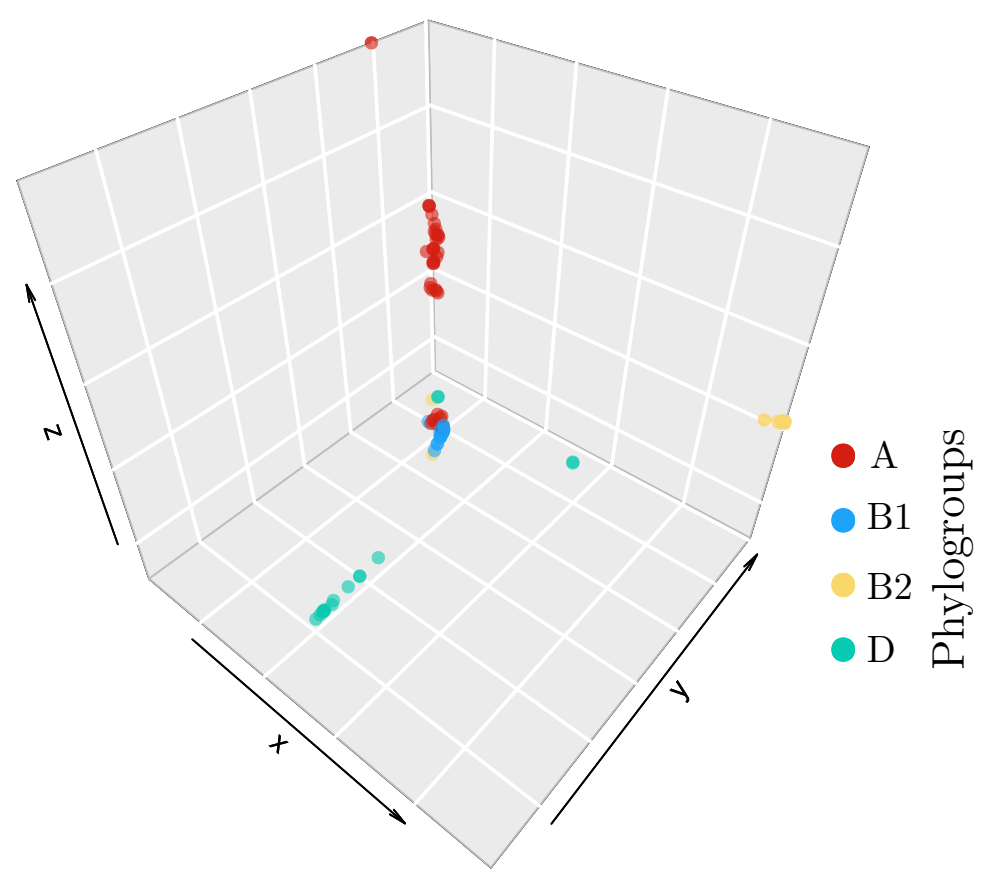

a) MDS Plot

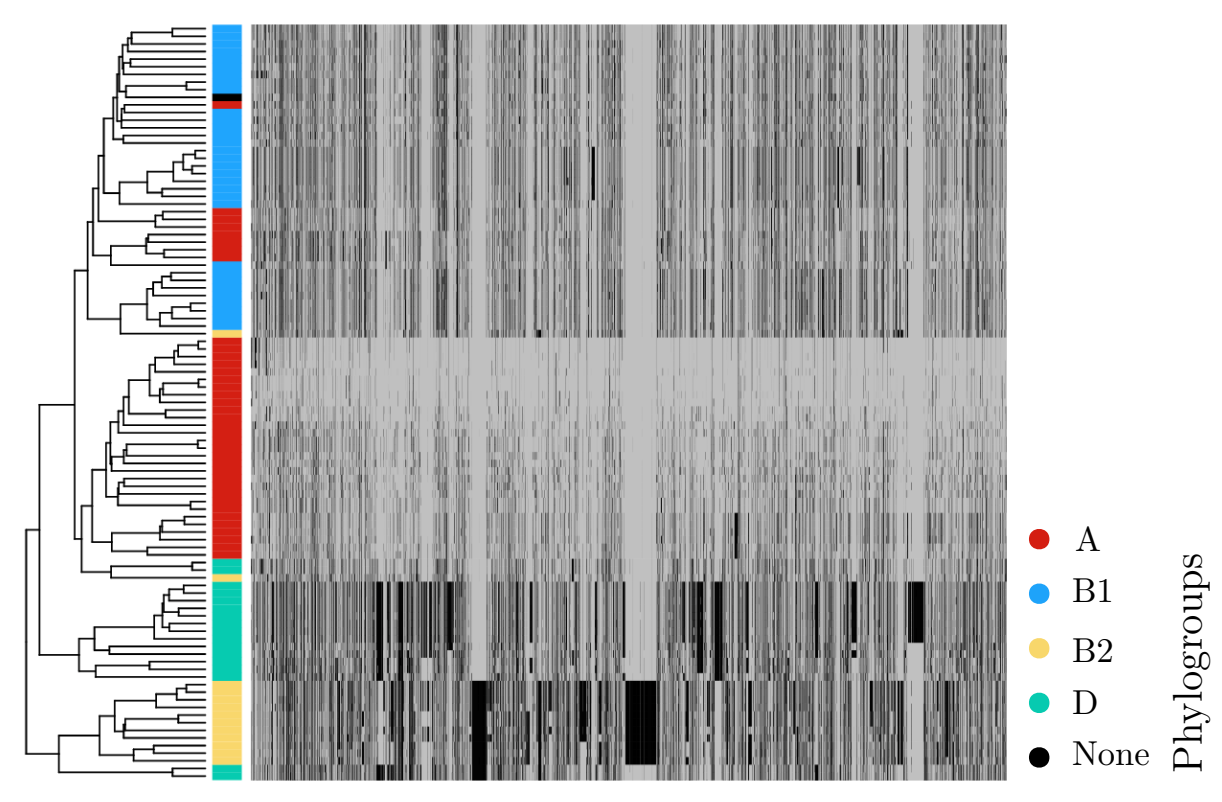

b) Hierarchical Clustering

Figure 6: a) Dimension reduction of isolates represented as high-dimensional vectors of all mutations. Four clusters are found, which reflect the population structure in the GWAS model and which broadly coincide with phylogroups A, B1, B2, and D. b) Same as a) but hierarchical clustering. Here, the presence of a mutation is shown by black and its absence by gray. 
bioRxiv preprint doi: https://doi.org/10.1101/2021.02.12.430739; this version posted February 13, 2021. The copyright holder for this preprint (which was not certified by peer review) is the author/funder, who has granted bioRxiv a license to display the preprint in perpetuity. It is made available under aCC-BY-ND 4.0 International license.

\section{Competing interests}

The authors declare that they have no competing interests.

\section{Author's contributions}

NM,TB, MS conceived the idea, TB contributed data, NM, AA, MS analysed data, NM, MS wrote the article.

\section{Acknowledgements}

We would like to thank Norhan Mahfouz, Eric Achatz, and Serena Caucci for an initial analysis of the data and valuable input and Magali De La Cruz Barron, Uli Klümper, Amay Ajaykumar Agrawal, Aldo Acevedo, Claudio Duran, and Mahmood Nazari for feedback. Funding of the ACRAS-R project is kindly acknowledged.

\section{Author details}

${ }^{1}$ Biotechnology Center (BIOTEC), Technische Universität Dresden, Tatzberg 47-49, 01307 Dresden, Germany. ${ }^{2}$ Institute of Hydrobiology, Technische Universität Dresden, Germany,

\section{References}

1. Emmerson, A., Jones, A.: The quinolones: decades of development and use. Journal of Antimicrobial Chemotherapy 51(suppl_1), 13-20 (2003)

2. Bisacchi, G.S.: Origins of the quinolone class of antibacterials: an expanded "discovery story" miniperspective. Journal of medicinal chemistry 58(12), 4874-4882 (2015)

3. Crumplin, G., Smith, J.: Nalidixic acid and bacterial chromosome replication. Nature 260(5552), 643-645 (1976)

4. Aldred, K.J., Kerns, R.J., Osheroff, N.: Mechanism of quinolone action and resistance. Biochemistry 53(10), 1565-1574 (2014)

5. Conrad, S., Saunders, J.R., Oethinger, M., Kaifel, K., Klotz, G., Marre, R., Kern, W.: gyra mutations in high-level fluoroquinolone-resistant clinical isolates of escherichia coli. Journal of Antimicrobial Chemotherapy 38(3), 443-456 (1996)

6. Hirschhorn, J.N., Daly, M.J.: Genome-wide association studies for common diseases and complex traits. Nature reviews genetics 6(2), 95 (2005)

7. Power, R.A., Parkhill, J., de Oliveira, T.: Microbial genome-wide association studies: lessons from human gwas. Nature reviews genetics 18(1), 41 (2017)

8. Chen, P.E., Shapiro, B.J.: The advent of genome-wide association studies for bacteria. Current opinion in microbiology 25, 17-24 (2015)

9. Lees, J.A., Vehkala, M., Välimäki, N., Harris, S.R., Chewapreecha, C. Croucher, N.J., Marttinen, P., Davies, M.R., Steer, A.C., Tong, S.Y., et al:: Sequence element enrichment analysis to determine the genetic basis of bacterial phenotypes. Nature communications 7, 12797 (2016)

10. Tenaillon, O., Skurnik, D., Picard, B., Denamur, E.: The population genetics of commensal escherichia coli. Nature Reviews Microbiology 8(3), 207-217 (2010)

11. Rasko, D.A., Rosovitz, M., Myers, G.S., Mongodin, E.F., Fricke, W.F., Gajer, P., Crabtree, J., Sebaihia, M., Thomson, N.R., Chaudhuri, R., et al:: The pangenome structure of escherichia coli: comparative genomic analysis of e. coli commensal and pathogenic isolates. Journal of bacteriology 190(20), 6881-6893 (2008)

12. Greenman, C., Stephens, P., Smith, R., Dalgliesh, G.L., Hunter, C. Bignell, G., Davies, H., Teague, J., Butler, A., Stevens, C., et al.: Patterns of somatic mutation in human cancer genomes. Nature 446(7132), 153-158 (2007)

13. Sharma, Y., Miladi, M., Dukare, S., Boulay, K., Caudron-Herger, M., Groß, M., Backofen, R., Diederichs, S.: A pan-cancer analysis of synonymous mutations. Nature communications 10(1), 1-14 (2019)

14. Kimchi-Sarfaty, C., Oh, J.M., Kim, I.-W., Sauna, Z.E., Calcagno, A.M., Ambudkar, S.V., Gottesman, M.M.: A" silent" polymorphism in the mdr1 gene changes substrate specificity. Science 315(5811), 525-528 (2007)
15. D'Costa, V.M., King, C.E., Kalan, L., Morar, M., Sung, W.W., Schwarz, C., Froese, D., Zazula, G., Calmels, F., Debruyne, R., et al.: Antibiotic resistance is ancient. Nature 477(7365), 457-461 (2011)

16. Berendonk, T.U., Manaia, C.M., Merlin, C., Fatta-Kassinos, D., Cytryn, E., Walsh, F., Bürgmann, H., Sørum, H., Norström, M., Pons, M.-N., et al.: Tackling antibiotic resistance: the environmental framework. Nature Reviews Microbiology 13(5), 310-317 (2015)

17. Mahfouz, N., Caucci, S., Achatz, E., Semmler, T., Guenther, S., Berendonk, T.U., Schroeder, M.: High genomic diversity of multi-drug resistant wastewater escherichia coli. Scientific reports $\mathbf{8}(1), 8928$ (2018)

18. McKenna, A., Hanna, M., Banks, E., Sivachenko, A., Cibulskis, K., Kernytsky, A., Garimella, K., Altshuler, D., Gabriel, S., Daly, M., et al.: The genome analysis toolkit: a mapreduce framework for analyzing next-generation dna sequencing data. Genome research 20(9), 1297-1303 (2010)

19. Van der Auwera, G.A., Carneiro, M.O., Hartl, C., Poplin, R., Del Angel, G., Levy-Moonshine, A., Jordan, T., Shakir, K., Roazen, D., Thibault, J., et al.: From fastq data to high-confidence variant calls: the genome analysis toolkit best practices pipeline. Current protocols in bioinformatics 43(1), 11-10 (2013)

20. Narasimhan, V., Danecek, P., Scally, A., Xue, Y., Tyler-Smith, C., Durbin, R.: Bcftools/roh: a hidden markov model approach for detecting autozygosity from next-generation sequencing data. Bioinformatics 32(11), 1749-1751 (2016)

21. Cingolani, P., Platts, A., Wang, L.L., Coon, M., Nguyen, T., Wang, L. Land, S.J., Lu, X., Ruden, D.M.: A program for annotating and predicting the effects of single nucleotide polymorphisms, snpeff: Snps in the genome of drosophila melanogaster strain w1118; iso-2; iso-3. Fly 6(2), 80-92 (2012)

22. Lees, J.A., Galardini, M., Bentley, S.D., Weiser, J.N., Corander, J.: pyseer: a comprehensive tool for microbial pangenome-wide association studies. Bioinformatics 34(24), 4310-4312 (2018)

23. Cook, D.E., Andersen, E.C.: Vcf-kit: assorted utilities for the variant call format. Bioinformatics 33(10), 1581-1582 (2017)

24. Purcell, S., Neale, B., Todd-Brown, K., Thomas, L., Ferreira, M.A., Bender, D., Maller, J., Sklar, P., De Bakker, P.I., Daly, M.J., et al.: Plink: a tool set for whole-genome association and population-based linkage analyses. The American journal of human genetics 81(3), 559-575 (2007)

25. Shin, J.-H., Blay, S., McNeney, B., Graham, J., et al.: Ldheatmap: an r function for graphical display of pairwise linkage disequilibria between single nucleotide polymorphisms. Journal of Statistical Software 16(3), 1-10 (2006)

26. Huerta-Cepas, J., Szklarczyk, D., Heller, D., Hernández-Plaza, A., Forslund, S.K., Cook, H., Mende, D.R., Letunic, I., Rattei, T., Jensen, L.J., et al.: eggnog 5.0: a hierarchical, functionally and phylogenetically annotated orthology resource based on 5090 organisms and 2502 viruses. Nucleic acids research 47(D1), 309-314 (2019)

27. Lord, D.M., Baran, A.U., Wood, T.K., Peti, W., Page, R.: Bdca, a protein important for escherichia coli biofilm dispersal, is a short-chain dehydrogenase/reductase that binds specifically to nadph. PloS one 9(9), 105751 (2014)

28. Ma, Q., Yang, Z., Pu, M., Peti, W., Wood, T.K.: Engineering a novel c-di-gmp-binding protein for biofilm dispersal. Environmental microbiology 13(3), 631-642 (2011)

29. Evans, D., Allison, D., Brown, M., Gilbert, P.: Susceptibility of pseudomonas aeruginosa and escherichia coli biofilms towards ciprofloxacin: effect of specific growth rate. Journal of Antimicrobial Chemotherapy 27(2), 177-184 (1991)

30. Høiby, N., Bjarnsholt, T., Givskov, M., Molin, S., Ciofu, O.: Antibiotic resistance of bacterial biofilms. International journal of antimicrobial agents 35(4), 322-332 (2010)

31. Manickam, Y., Chaturvedi, R., Babbar, P., Malhotra, N., Jain, V., Sharma, A.: Drug targeting of one or more aminoacyl-trna synthetase in the malaria parasite plasmodium falciparum. Drug discovery today 23(6), 1233-1240 (2018)

32. Agarwal, V., Nair, S.K.: Aminoacyl trna synthetases as targets for antibiotic development. MedChemComm 3(8), 887-898 (2012)

33. Garoff, L., Huseby, D.L., Praski Alzrigat, L., Hughes, D.: Effect of aminoacyl-trna synthetase mutations on susceptibility to ciprofloxacin 
bioRxiv preprint doi: https://doi.org/10.1101/2021.02.12.430739; this version posted February 13, 2021. The copyright holder for this preprint (which was not certified by peer review) is the author/funder, who has granted bioRxiv a license to display the preprint in perpetuity. It is made Malekian et al. available under aCC-BY-ND 4.0 International license.

Page 13 of 13

in escherichia coli. Journal of Antimicrobial Chemotherapy 73(12), 3285-3292 (2018)

34. Abergel, C., Monchois, V., Byrne, D., Chenivesse, S., Lembo, F., Lazzaroni, J.-C., Claverie, J.-M.: Structure and evolution of the ivy protein family, unexpected lysozyme inhibitors in gram-negative bacteria. Proceedings of the National Academy of Sciences 104(15), 6394-6399 (2007)

35. Ruiz, N., Gronenberg, L.S., Kahne, D., Silhavy, T.J.: Identification of two inner-membrane proteins required for the transport of lipopolysaccharide to the outer membrane of escherichia coli. Proceedings of the National Academy of Sciences 105(14), 5537-5542 (2008)

36. Luo, H., Lin, Y., Liu, T., Lai, F.-L., Zhang, C.-T., Gao, F., Zhang, R. Deg 15, an update of the database of essential genes that includes built-in analysis tools. Nucleic Acids Research (2020)

37. Ma, Q., Zhang, G., Wood, T.K.: Escherichia coli bdca controls biofilm dispersal in pseudomonas aeruginosa and rhizobium meliloti. BMC Research Notes 4(1), 447 (2011)

38. Stoppe, N.d.C., Silva, J.S., Carlos, C., Sato, M.I., Saraiva, A.M., Ottoboni, L.M., Torres, T.T.: Worldwide phylogenetic group patterns of escherichia coli from commensal human and wastewater treatment plant isolates. Frontiers in microbiology 8, 2512 (2017)

39. Hurdle, J.G., O'Neill, A.J., Chopra, I.: Prospects for aminoacyl-trna synthetase inhibitors as new antimicrobial agents. Antimicrobial agents and chemotherapy 49(12), 4821-4833 (2005) 